\title{
LINFADENOCROMATOGRAFIA PERIGÁSTRICA COM CARBONO ATIVADO (CH40): ESTUDO EXPERIMENTAL
}

\author{
Perigastric lymphadenochromatography with activated carbon (CH40): \\ experimental study
}

\author{
Minao OKAWA ${ }^{2}$, Luciano OKAWA ${ }^{2}$, Hugo MEISTER ${ }^{1}$
}

ABCDDV/555

Okawa M, Okawa L, Meister H. Linfadenocromatografia perigástrica com carbono ativado (CH40): estudo experimental. ABCD Arq Bras Cir Dig 2007;20(3):184-8.

RESUMO - Racional - Procedimento cirúrgico bem instituído e planejado constitui ainda a única terapêutica efetiva para o câncer gástrico. Entretanto, os resultados de cura não têm melhorado significativamente nas últimas décadas, principalmente no ocidente. O principal benefício de introdução endoscópica de corantes na submucosa gástrica está na facilitação de linfadenectomias regradas, podendo facilitar a localização acurada dos linfonodos. Além disso, a linfadenocromatografia possibilita futuras pesquisas para a deteç̧ão do linfonodo sentinela e sua aplicabilidade racional e seletiva na indicação precisa da linfadenectomia, principalmente dos grupos de linfonodos de ressecção mais trabalhosa e com maiores possibilidades de complicações. A linfadenocromatografia com carbono ativado (CH40) foi analisada neste estudo experimental (fase 1). Objetivos - Testar a efetividade e inocuidade da linfadenocromatografia com CH40, em três espécies animais diferentes. Métodos - Como preconizado pela Associação Brasileira de Pesquisas Clínicas (Resoluções n ${ }^{\circ}$ 196/96 e 251/97, CNS/MS), foram utilizados três espécies diferentes de animais: 3 cães, 10 coelhos e 20 ratos. Em todos os cães, a introdução de CH40 na camada submucosa de estômago foi realizada através da endoscopia gástrica e utilizando, cateter de polietileno agulhado, usualmente empregado na esclerose de varizes esofageanas. O período de observação variou de 43 a 125 dias, quando o procedimento cirúrgico foi realizado. Em todos os coelhos e ratos a introdução de CH40 foi realizada através de agulha hipodérmica e seringa insulínica, na região subcutânea inguinal. O período de observação foi de 102 a 105 dias e 110 a 122 dias, respectivamente. Resultados - Nos períodos de observação, desde a injeção até a eutanásia, não se detectou em nenhum animal efeito colateral ou óbito. Nos cães, linfonodos perigástricos foram corados pelo $\mathrm{CH} 40$. Em todos os coelhos e ratos, o tecido celular subcutâneo da região inguinal foi fortemente corado de preto. Também, em todos ratos, linfonodos da região aórtica abdominal foram tingidos pelo CH40. Conclusãa - A linfadenocromatografia com CH40 não mostrou toxicidade nas três espécies de animais estudadas. O carbono ativado foi efetivo no tingimento de linfonodos neste estud

DESCRITORES - Carbono. Neoplasias gástricas. Cromatografia. Linfonodos.

\section{INTRODUÇÃO}

Câncer gástrico é neoplasia maligna, originada no estômago, decorrente de sucessivas mutações genéticas na célula de origem devido a fatores intrínsecos e/ou extrínsecos, sendo de maior prevalência a do tecido glandular, denominado de adenocarcinoma ${ }^{1,8}$.

Há apreciável diminuição da incidência de neoplasia gástrica, principalmente dos adenocarcinomas localizados no estômago distal, nos países da Europa Ocidental e nos Estados Unidos, contrariamente a maior incidência no nosso meio. A variação da incidência do câncer gástrico nestes diferentes países sugere que os fatores de risco devam ser de origem ambiental, destacando-se os fatores dietéticos.

Operação bem instituída e planejada constitui ainda a única terapêutica efetiva, porém os resultados de cura não têm melhorado significativamente nas últimas quatro décadas, principalmente no mundo ocidental ${ }^{1,2}$.

O esforço cirúrgico para a melhoria prognóstica do câncer gástrico, por meio das operações e das linfadenecto-

Trabalho realizado no Departamento de Medicina, Disciplina de Técnica Operatória, Universidade Estadual de Maringá - UEM1 e Hospital Paraná ${ }^{1}$, Maringá, PR, Brasil.

Endereço para corespondência: Minao Okawa, e-mail: okawa@brturbo.com.br mias regradas, adequadamente indicadas em sua extensão e magnitude e na radicalidade operatória bem conduzida, é sentido em vários centros de excelência no tratamento dessa afecção.

As injeções intra-operatórias de corantes são realizadas para visibilização dos grupos linfonodais para ressecções, porém não é de prática habitual no nosso meio e apresenta certas inconveniências como a precisa introdução do produto químico no linfonodo da região requerida e dificuldades do procedimento nos obesos. Os corantes mais tradicionais ainda em uso são os denominados corantes vitais, como azul-patente.

A medicina nuclear com tecnécio 99, ligado a um colóide e injetado em determinada área tumoral e captado pelos linfonodos marcados, com gama probe, mesmo com variantes técnicas e associações, tem-se mostrado útil na realização da linfografia para detecção de linfonodos, independentemente de presença metastática neles. Contrastes radiológicos e tomográficos também foram empregados ${ }^{1,2,3,8}$.

A principal contribuição para a melhoria no tratamento cirúrgico dos portadores de câncer gástrico seria a eficácia das linfadenectomias regradas, sensibilizadas pela visibilidade mais patente mediante o tingimento inócuo dos linfáticos e dos linfonodos das cadeias perigástricas, com material corante introduzido por via endoscópica na 
camada submucosa do estômago. Além disso, a linfadenocromatografia possibilita futuras pesquisas para a deteç̧ão do linfonodo sentinela e sua aplicabilidade racional e seletiva na indicação precisa da linfadenectomia, principalmente dos grupos de linfonodos de ressecção mais trabalhosa e com maiores possibilidades de complicações ${ }^{5,8}$.

Apesar de ser utilizado para linfadenocromatografia perigástrica em países orientais, poucas publicações em língua inglesa citam o CH40. No Brasil, também poucos centros já utilizaram o carbono ativado para tingimento linfonodal.

Assim, o objetivo deste trabalho foi testar a efetividade e inocuidade da linfadenocromatografia com $\mathrm{CH} 40$, em três espécies animais diferentes.

\section{MÉTODOS}

Esta pesquisa obedeceu as seguintes resoluções: do Ministério da Saúde Resolução no 196/96, 10 de outubro, do CNS/ MS; Resolução n ${ }^{\circ}$ 251/97, de 5 de agosto, do CNS/MS e em Normas de Pesquisa com Novos Fármacos, Medicamentos, Vacinas e Testes Diagnósticos envolvendo seres humanos?.

Foram utilizados três cães da raça Beagle, espécie canídeos canis familiaris, todos machos com idade de dois anos; 10 coelhos, Oryctolaus cuniculus, albinos da linhagem Nova Zelândia, sendo cinco machos e cinco fêmeas, com idade de 4 meses, e 20 ratos da espécie Rattus novergicus, da linhagem Wistar, sendo 10 machos e 10 fêmeas com idade de 3 meses.

O produto químico empregado na pesquisa foi um derivado de carbono 40 ativado, denominado CH40. Este consiste em solução obtida por meio de composições de carbono ativado 40, polivinilpirrolidona (PVP) e solução salina. As proporções utilizadas foram de $50 \mathrm{mg} / \mathrm{mL}$ de carbono ativado C40, $20 \mathrm{mg} / \mathrm{mL}$ de PVP e solução salina para que depois de batidos em três cilindros, tornar o carbono ativado em uma suspensão estável, com partículas de diâmetro em torno de $150 \mathrm{~nm}$. O carbono ativado C40 foi fornecido pela Mitsubishi Co. Ltd., Tokyo, Japan e PVP, K-30, pela Nakarai Chemicals Co., Ltd., Kyoto, Japan.

Todos os cães foram submetidos à endoscopia digestiva alta. A introdução de CH40 foi realizada através de cateter de polietileno agulhado, em quantidade de $0,5 \mathrm{~mL}$, em cada punção nas paredes anterior e posterior, na pequena e grande curvatura de antro e corpo gástrico, totalizando $4,0 \mathrm{~mL} \mathrm{em}$ cada procedimento (Figura 1). Os cães ficaram confinados e enjaulados, após o procedimento, sendo observados diariamente, por 43, 80 e 125 dias, respectivamente, até a eutanásia.

Os 10 coelhos receberem $1 \mathrm{~mL}$ de CH40, através de agulha e seringa hipodérmicas, no tecido subcutâneo inguinal. Todos os coelhos permaneceram separados individualmente em gaiolas e mantidos em locais apropriados e observados também diariamente, de 102 a 105 dias, até a eutanásia.

Os 20 ratos receberem $1 \mathrm{~mL}$ de CH40, através de agulha e seringa hipodérmicas, no tecido subcutâneo inguinal. Foram observados por 110 a 122 dias, até a eutanásia.

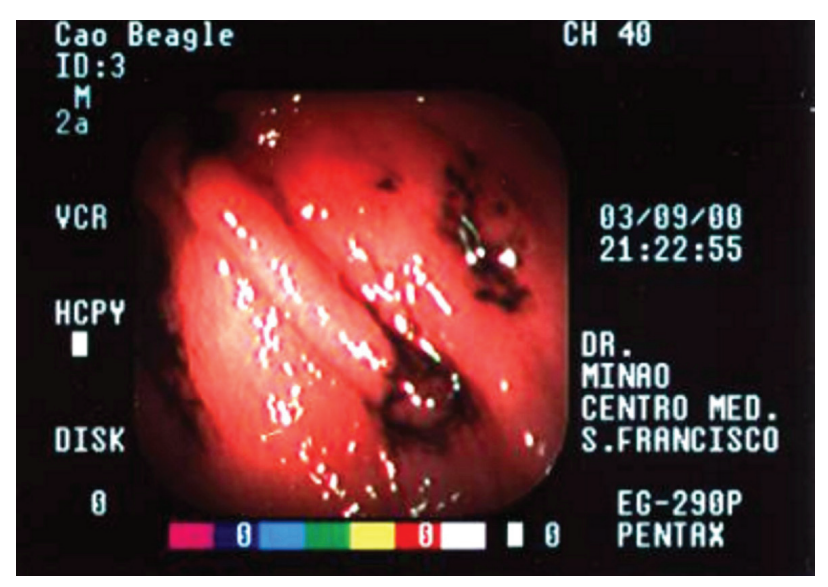

FIGURA 1 - Mucosa gástrica de cão corada com CH40

\section{RESULTADOS}

\section{Em cães}

Na observação clínica de até 125 dias após a introdução de $\mathrm{CH} 40$, na camada submucosa gástrica dos referidos animais, não apresentou alteração de conduta, alteração do estado físico e óbito. Todos tiveram seu peso aumentado, constatando-se a inocuidade do corante em experimentação nesse animal (Tabela 1).

TABELA 1 - Cães: pesos no início do estudo e na eutanásia

\begin{tabular}{lcc}
\hline Cães & Pesos no início do estudo & Pesos na eutanásia \\
\hline $\mathrm{N}^{\mathrm{o}} 1$ & $10,500 \mathrm{~kg}$ & $11,200 \mathrm{~kg}$ \\
$\mathrm{~N}^{\mathrm{o}} 2$ & $11,100 \mathrm{~kg}$ & $11,600 \mathrm{~kg}$ \\
$\mathrm{~N}^{\mathrm{o}} 3$ & $10,500 \mathrm{~kg}$ & $11,200 \mathrm{~kg}$ \\
Peso médio & $10,700 \mathrm{~kg}$ & $11,333 \mathrm{~kg}$ \\
\hline
\end{tabular}

Verificou-se também, apesar da escassez dos linfonodos perigástricos nesses cães, a efetividade da coloração linfonodal pelo $\mathrm{CH} 40$ nas peças anatômicas gastroduodenaisepiplóicas ressecadas na eutanásia (Figuras 2 e 3).

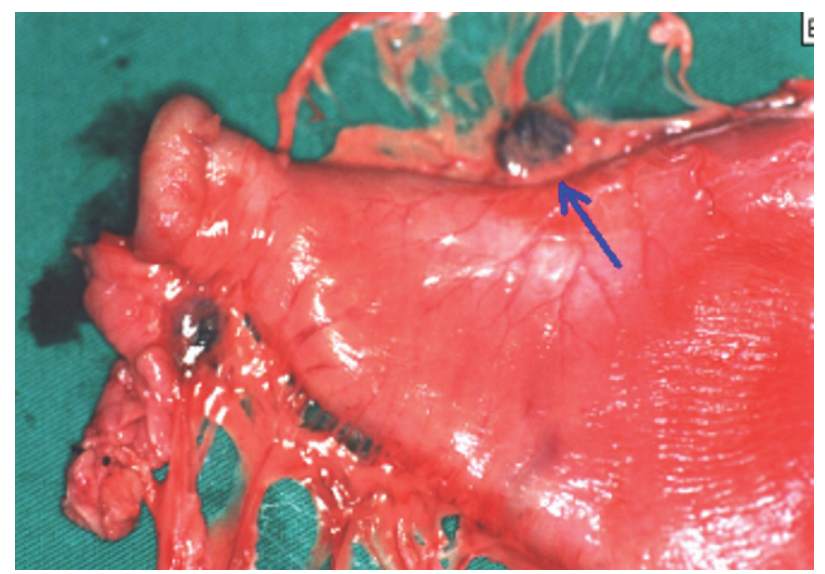

FIGURA 2 - Cães: linfonodo da pequena curvatura corado pelo $\mathrm{CH} 40$ 


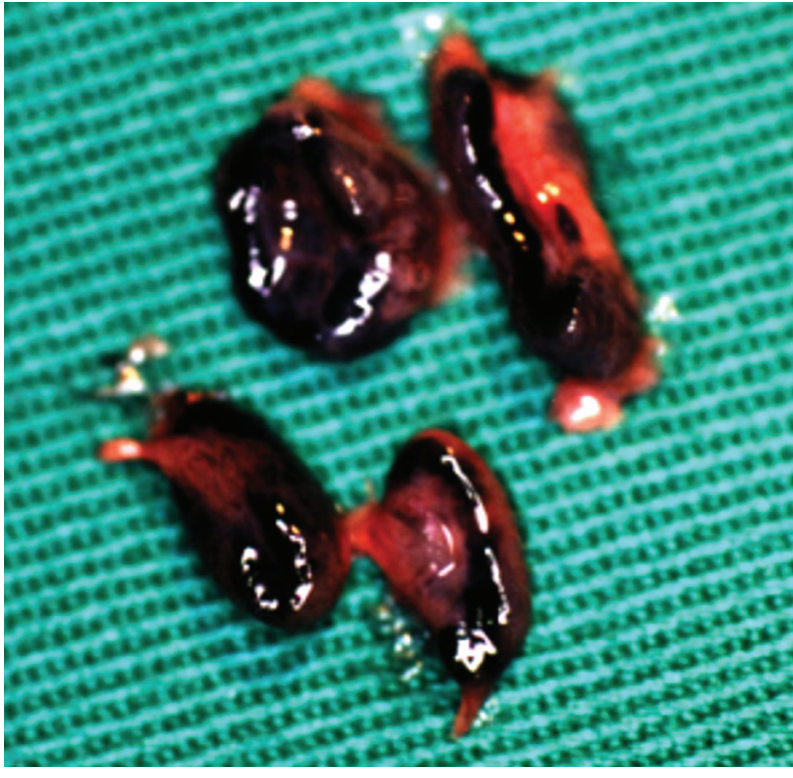

FIGURA 3 - Cães: linfonodos perigástricos dissecados, corados pelo $\mathrm{CH} 40$.

\section{Em coelhos}

Na observação clínica de todos os coelhos até 105 dias após injeção subcutânea de CH40 na região inguinal não houve nenhum óbito, nem foram observadas alterações físicas ou de conduta, senão aumento de peso na maioria absoluta (Tabela 2). Constatou-se a inocuidade do $\mathrm{CH} 40$ nesses animais.

TABELA 2 - Coelhos: pesos no início do estudo e na eutanásia

\begin{tabular}{lcc}
\hline Coelhos & Pesos no início do estudo & Pesos na eutanásia \\
\hline $\mathrm{N}^{\circ} 01$ & $1,800 \mathrm{~kg}$ & $1,850 \mathrm{~kg}$ \\
$\mathrm{~N}^{\circ} 02$ & $2,200 \mathrm{~kg}$ & $2,500 \mathrm{~kg}$ \\
$\mathrm{~N}^{\circ} 03$ & $2,000 \mathrm{~kg}$ & $1,800 \mathrm{~kg}$ \\
$\mathrm{~N}^{\circ} 04$ & $2,100 \mathrm{~kg}$ & $3,200 \mathrm{~kg}$ \\
$\mathrm{~N}^{\circ} 05$ & $2,450 \mathrm{~kg}$ & $2,800 \mathrm{~kg}$ \\
$\mathrm{~N}^{\circ} 06$ & $2,100 \mathrm{~kg}$ & $2,300 \mathrm{~kg}$ \\
$\mathrm{~N}^{\circ} 07$ & $2,200 \mathrm{~kg}$ & $3,270 \mathrm{~kg}$ \\
$\mathrm{~N}^{\circ} 08$ & $2,500 \mathrm{~kg}$ & $3,650 \mathrm{~kg}$ \\
$\mathrm{~N}^{\circ} 09$ & $2,250 \mathrm{~kg}$ & $3,320 \mathrm{~kg}$ \\
$\mathrm{~N}^{\circ} 10$ & $2,500 \mathrm{~kg}$ & $2,800 \mathrm{~kg}$ \\
Peso médio & $2,210 \mathrm{~kg}$ & $2,749 \mathrm{~kg}$ \\
\hline
\end{tabular}

Não se verificou tingimento de nenhum linfonodo na região para-aórtica intra-abdominal em todos os coelhos, e sim forte coloração enegrecida subcutânea na região inguinal, local de introdução de CH40 (Figura 4).

\section{Em ratos}

Na observação clínica de todos os ratos, até 112 dias após injeção subcutânea de $\mathrm{CH} 40$ na região inguinal, não houve nenhum óbito, nem foram observadas alterações de conduta ou físicas, senão aumento de peso na maioria

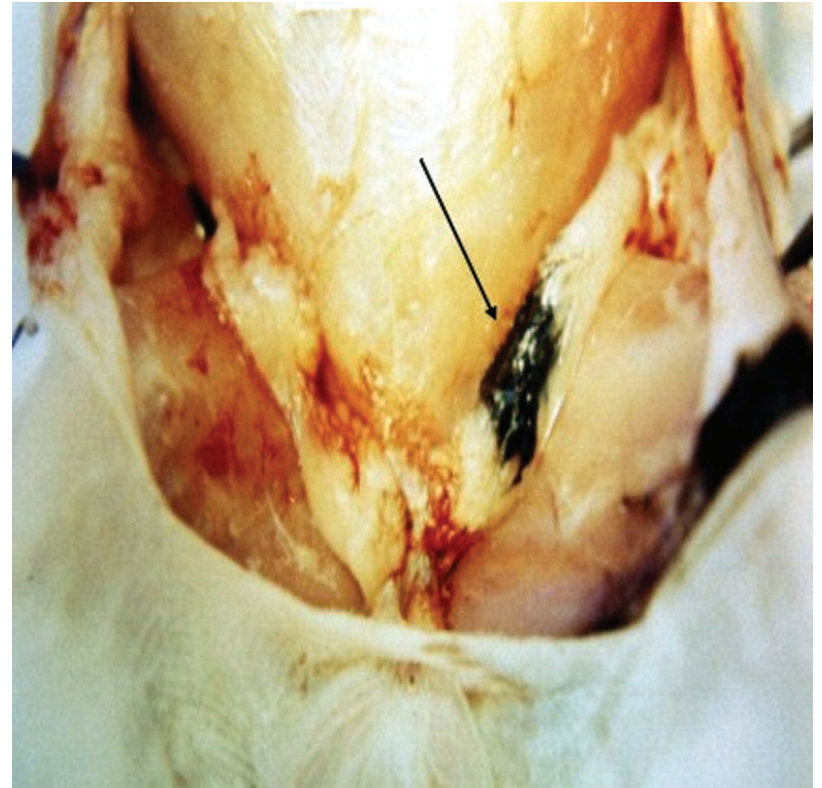

FIGURA 4 - Coelhos: tecido subcutâneo da região inguinal esquerda corado pelo $\mathrm{CH} 40$

absoluta no dia da eutanásia (Tabela 3), constatando-se assim a inocuidade do produto de experimentação, nesse tipo de animal.

TABELA 3 - Ratos: pesos no início do estudo e na eutanásia.

\begin{tabular}{|c|c|c|}
\hline Ratos & Pesos no início do estudo & Pesos na eutanásia \\
\hline $\mathrm{N}^{\mathrm{o}} 01$ & $305 \mathrm{~g}$ & $320 \mathrm{~g}$ \\
\hline $\mathrm{N}^{\mathrm{o}} 02$ & $300 \mathrm{~g}$ & $305 \mathrm{~g}$ \\
\hline $\mathrm{N}^{\mathrm{o}} 03$ & $305 \mathrm{~g}$ & $315 \mathrm{~g}$ \\
\hline $\mathrm{N}^{\mathrm{o}} 04$ & $310 \mathrm{~g}$ & $315 \mathrm{~g}$ \\
\hline $\mathrm{N}^{\circ} 05$ & $305 \mathrm{~g}$ & $305 \mathrm{~g}$ \\
\hline $\mathrm{N}^{\mathrm{o}} 06$ & $350 \mathrm{~g}$ & $370 \mathrm{~g}$ \\
\hline $\mathrm{N}^{\circ} 07$ & $400 \mathrm{~g}$ & $405 \mathrm{~g}$ \\
\hline $\mathrm{N}^{\mathrm{o}} 08$ & $310 \mathrm{~g}$ & $305 \mathrm{~g}$ \\
\hline $\mathrm{N}^{\mathrm{o}} 09$ & $365 \mathrm{~g}$ & $390 \mathrm{~g}$ \\
\hline $\mathrm{N}^{\mathrm{o}} 10$ & $355 \mathrm{~g}$ & $355 \mathrm{~g}$ \\
\hline $\mathrm{N}^{\circ} 11$ & $340 \mathrm{~g}$ & $370 \mathrm{~g}$ \\
\hline $\mathrm{N}^{\mathrm{o}} 12$ & $350 \mathrm{~g}$ & $425 \mathrm{~g}$ \\
\hline $\mathrm{N}^{\mathrm{o}} 13$ & $350 \mathrm{~g}$ & $350 \mathrm{~g}$ \\
\hline $\mathrm{N}^{\circ} 14$ & $375 \mathrm{~g}$ & $385 \mathrm{~g}$ \\
\hline $\mathrm{N}^{\circ} 15$ & $320 \mathrm{~g}$ & $315 \mathrm{~g}$ \\
\hline $\mathrm{N}^{\mathrm{o}} 16$ & $310 \mathrm{~g}$ & $325 \mathrm{~g}$ \\
\hline $\mathrm{N}^{\mathrm{o}} 17$ & $300 \mathrm{~g}$ & $310 \mathrm{~g}$ \\
\hline $\mathrm{N}^{\mathrm{o}} 18$ & $315 \mathrm{~g}$ & $320 \mathrm{~g}$ \\
\hline $\mathrm{N}^{\circ} 19$ & $310 \mathrm{~g}$ & $315 \mathrm{~g}$ \\
\hline $\mathrm{N}^{\mathrm{o}} 20$ & $320 \mathrm{~g}$ & $335 \mathrm{~g}$ \\
\hline Peso médio & $329,75 \mathrm{~g}$ & $341,75 \mathrm{~g}$ \\
\hline
\end{tabular}

Houve forte coloração enegrecida de tingimento subcutâneo na região inguinal, local de introdução de CH40. Importa salientar que todos os 20 ratos tiveram linfonodos intra-abdominais, da região para-aórtica fortemente enegrecidos (Figura 5). 


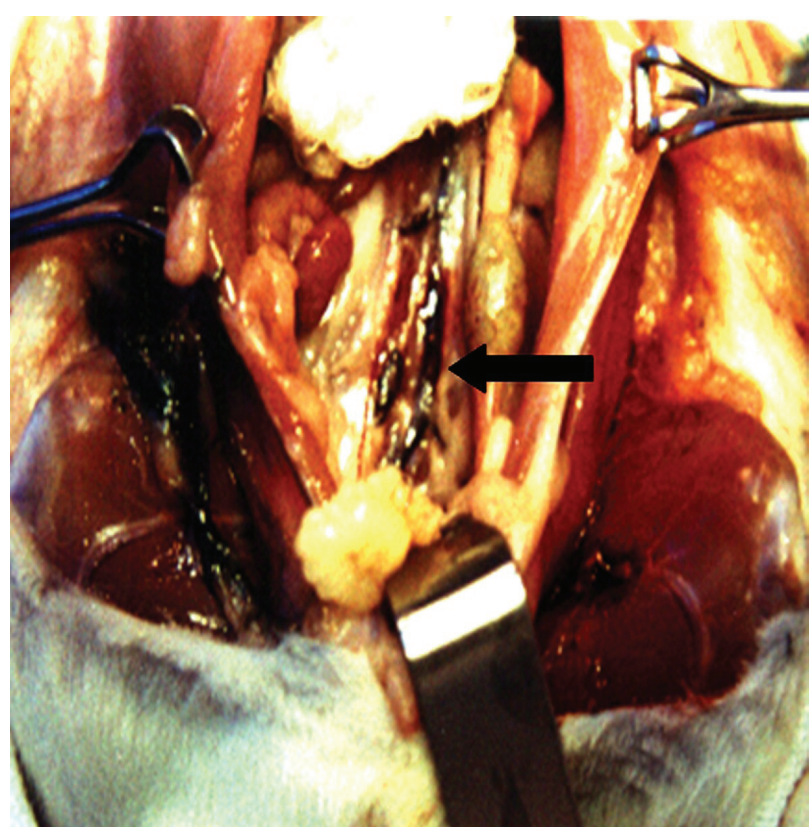

FIGURA 5 - Ratos: linfonodos para-aórticos corados pelo $\mathrm{CH} 40$.

\section{DISCUSSÃO}

A coloração linfonodal perigástrica e sua aplicação nas linfadenectomias iniciaram-se em 1950, quando Weinberg e Greaney utilizaram um corante químico denominado "pontaine sky blue". Desde então, vários corantes, contrastantes e radioisótopos foram utilizados de diversas maneiras na intenção de tingir linfonodos perigástricos, com objetivo de identificar grupos constituintes para ressecção ou para estudo de drenagem linfática de uma determinada região ${ }^{10}$.

A utilização de CH40 iniciou-se em 1983 com Hagiwara ${ }^{1}$, no Hospital de Kyoto Prefectural University of Medicine do Japão, quando adsorveu a mitomicina $\mathrm{C}$ em carbono ativado $\mathrm{CH} 40$, na pesquisa de nova forma e dosagem quimioterápica na neoplasia maligna do estômago.

No presente estudo, carbono ativado (CH40) foi analisado em relação a sua inocuidade e difusibilidade através de tecidos, e principalmente de linfáticos, em observância às normas de pesquisas clínicas oriundas em forma de resoluções do Ministério da Saúde, que regulamentam a realização em três animais distintos, sendo que em pelo menos um o procedimento deve ser semelhante ao da segunda fase, em seres humanos. O uso desse material deveu-se ao tamanho pigmentar muito reduzido, que lhe confere maior possibilidade de coloração de linfonodos perigástricos, quando injetado na camada submucosa gástrica por meio da endoscopia ${ }^{4,5}$.

O corante pesquisado e utilizado foi um derivado do carbono 40 , com partículas em torno de $20 \mathrm{~nm}, 50 \mathrm{mg} / \mathrm{mL}$, combinado com $20 \mathrm{mg} / \mathrm{mL}$ de polivinilpirrolidona (PVP, K- 30 Nakarai Chemicals Co., Ltda., Kyoto, Japan) e misturados em solução salina, batidos em cilindros, transformando o carbono em forma de suspensão líquida, preta, com partículas uniformemente menores que $700 \mathrm{~nm}$, em média $150 \mathrm{~nm}$ (Hagiwara et al., 1992) 7 .

Denominou-se de "ativado" porque se torna mais ativo por ação química e física reacional, conferindo-lhe maior poder de adsorção devido à grande superfície porosa, com possibilidade de impregnação nessa superfície por aderência atrativa, e aumento da difusibilidade do composto de carbono ativado, pelo fato de as partículas serem muito pequenas $^{5,6}$.

Esse corante foi idealizado por Hagiwara e publicado em 1983 em língua japonesa, e junto com colaboradores em 1992 em língua inglesa ${ }^{2,7}$. É considerado corante permanente.

Para sua utilização, o CH40 deve ser esterilizado em autoclave, sendo tomados todos os cuidados assépticos no seu emprego, não podendo ser guardado em geladeira ou em condições semelhantes, mas em temperatura ambiental. Quando o CH40 é re-esterilizado em altas temperaturas ou mantido sob condições geladas, as partículas de carbono se aglomeram e o tamanho das partículas da suspensão volta a ser de tamanho maior, com diminuição da possibilidade de tingimento linfonodal.

Apesar de ser utilizado há vários anos em países orientais, poucas publicações em língua inglesa citam o $\mathrm{CH} 40$ como corante em linfadenocromatografias. Em nosso país, este corante é pouco conhecido e utilizado.

Nenhuma pesquisa relata endoscopia em cães e introdução de CH40 na submucosa gástrica para obtenção de coloração linfonodal perigástrica. Neste estudo, nenhum cão apresentou efeitos colaterais ou óbito após receber carbono ativado através de endoscopia digestiva alta. Linfonodos perigástricos corados pelo $\mathrm{CH} 40$ foram observados em todos os cães.

Em coelhos, a introdução de $\mathrm{CH} 40$ foi realizada na região subcutânea da prega inguinal. Não houve nenhum óbito, nem foram observados efeitos colaterais. Todos, após eutanásia, foram dissecados e examinados os locais da introdução do $\mathrm{CH} 40$ e a cavidade abdominal para exame da região para-aórtica abdominal. Não foram observados linfonodos corados nessa região. Em coelhos, a utilização de CH40 também não é referida na literatura, como também as injeções subcutâneas de CH40 na região inguinal, o qual tinge de maneira intensa o tecido celular subcutâneo do local mencionado.

Em ratos, a introdução de $\mathrm{CH} 40$ foi também realizada na região subcutânea da prega inguinal, tingindo intensamente o tecido subcutâneo. Não houve óbito nem efeitos adversos observados. Todos, após eutanásia, foram dissecados e examinados os locais da introdução de $\mathrm{CH} 40$ e a cavidade abdominal para exame da região para-aórtica abdominal, igual a dos coelhos. Todos os ratos tiveram os linfonodos para-aórticos corados pelo CH40.

Hagiwara et al., em 1992 ${ }^{7}$, introduziram CH40 na prega subcutânea da pata traseira direita de ratos, com coloração do linfonodo poplíteo em $100 \%$, no ilíaco de $85 \%$ e no para-aórtico de $65 \%$, decorridos oito minutos da introdução até a eutanásia.

Na presente pesquisa, em 20 ratos, com introdução de CH40 através da injeção subcutânea na região inguinal, 
decorridos entre 110 a 122 dias de observação clínica até a eutanásia, a coloração linfonodal para-aórtico foi de $100 \%$.

O presente estudo experimental de tingimento linfonodal está intencionalmente baseado na orientação ao cirurgião quando da identificação, dissecção e ressecção dos grupos linfonodais com possíveis metástases no câncer gástrico.

\section{CONCLUSÕES}

O procedimento com $\mathrm{CH} 40$ em três espécies animais submetidas à experimentação não mostrou toxicidade, durante as observações clínicas. O carbono ativado foi efetivo no tingimento de linfonodos neste estudo.

Okawa M, Okawa L, Meister H. Perigastric lymphadenochromatography with activated carbon (CH40): experimental study. ABCD Arq Bras Cir Dig 2007;20(3):184-8.

ABSTRACT - Background - Well instituted and planned surgical procedures are still the only effective therapy for gastric cancer. However, results of cure have not significantly improved in the last decades, especially in the western world. The main benefit of the introduction of dyes and colouring in gastric submucosa through endoscopy, is to facilitate moderate lymphadectomy, making it easier to accurately locate lymph nodes. Besides this, lymphadenochromatography makes future research possible in detecting sentinel lymph nodes and its rational and selective applicability in precise lymphadectomy indication, especially in groups submitted to more complicated lymph node ressection which have increased possibility of complications. Carbon actived (CH40) lymphadenochromatography was analysed in this experimental study (phase 1). Aim - To test the effectiveness and innocuity of lymphadenochromatography with CH40, in three different animal species. Methods - As established by the Brazilian Association of Clinical Research (Resolutions n ${ }^{\circ}$ 196/96 e 251/97, CNS/MS), three different animal species were used: three dogs, 10 rabbits and 20 rats. In all of the dogs, the introduction of $\mathrm{CH} 40$ in the submucosa layer of the stomach was performed using gastric endoscopy, as well as needle polyethylene catheters, usually employed in sclerosis of esophageal varicosis. The observation period varied between 43 to 125 days, when surgical procedures were performed. In all rabbits and rats, the introduction of $\mathrm{CH} 40$ was made through hypodermic needle and insulinic syringe in the subcutaneous inguinal region. The observation period was between 102 through 105 days and 110 to 122 days, respectively. Results - During the observation periods, from injection to euthanasia, no side effects or death was detected in any animal. In the dogs, perigastric lymph nodes were dyed using $\mathrm{CH} 40$. In all rabbits and rats, the subcutaneous cellular tissue of the inguinal region was dyed black strongly. Also, in all rats, lymph nodes of the abdominal aortic region were colored using CH40. Conclusions - Lymphadenochromatography with CH40 did not demonstrate toxicity in the three animal species studied. Actived carbon was effective in the coloring of lymph nodes in this study.

HEADINGS - Carbon. Stomach neoplasms. Chromatography. Lymph nodes.

\section{REFERÊNCIAS}

1. Hagiwara A. Mitomicym $\mathrm{C}$ adsorbed to activated carbon particles as a new drug dosage form for cancer chemotherapy. Akita J Med. 1983;10:187-229.

2. Hagiwara A, Takahashi T, Lee R. In vitro examination of mitomycin C adsorbed to small sized activated carbon particle. Akita J Med. 1984;10:419-22.

3. Hagiwara A, Ahn T, Ueda T, Takahashi T. Anticancer agents adsorbed by activated carbon particles, a new form of dosage enhancing efficacy on lymphnodal metastases. Anticancer Res. 1986;6:1005-8.

4. Hagiwara A, Ueda T, Sato S. Reduced toxicity of mitomycin C in a new dosage form - mitomycin $\mathrm{C}$ adsorbed on activated carbon particles. Japan Red Cross Medical J. 1986;38:17-20.

5. Hagiwara A, Takahashi T, Ueda T. Activated carbon particles as anti-cancer drug carrier into regional lymph nodes. Anticancer Design. 1987;1:313-21.

6. Hagiwara A, Takahashi T, Oku N. Cancer chemotherapy administered by activated carbon particles and liposomes. Crit Rev Oncol Hematol. 1989;9:31950
7. Hagiwara A, Takahashi T, Sawai K, Iwamoto A, Shimotsuma M, Yoneyama C, Seiki K, Itoh M, Sasabe T, Lee M. Lymph nodal staining with newer carbon particle suspensions compared with India ink: experimental and clinical observations. Lymphology. 1992:25;84-9.

8. Okawa M. Linfadenocromatografia perigástrica com carbono ativado $(\mathrm{CH} 40)$ - estudo experimental e clínico [tese]. Curitiba: Universidade Federal do Paraná; 2002.

9. Resolução CNS N ${ }^{\circ}$ 251/97, de 07 de Agosto de 1997, homologado nos termos do Decreto de Delegação de Competência de 12 de Novembro de 1991, pelo Carlos César De Albuquerque - Presidente do Conselho e Ministro do Estado da Saúde. Diário Oficial da União. 23/09/97; Seção I, p.21117.

10. Weinberg J, Greaney EM. Identification of regional lymph nodes by means of a vital staining dye during surgery of gastric cancer. Surg Gynecol Obstet. 1952;90:561-7.

Conflito de interesse: não há

Fonte financiadora: não há

Recebido para publicação em: 03/04/2007 Aceito para publicação em: 20/05/2007 